\title{
Scavenger receptor class B type $I$ is a multiligand HDL receptor that influences diverse physiologic systems
}

\author{
Monty Krieger \\ Department of Biology, Massachusetts Institute of Technology, Room 68-483, Cambridge, Massachusetts 02139, USA. \\ Phone: (617) 253-6793; Fax: (617) 258-5851; E-mail: krieger@mit.edu.
}

J. Clin. Invest. 108:793-797 (2001). DOI:10.1172/JCI200114011.

Scavenger receptors (SRs) are defined as cell surface membrane proteins that bind chemically modified lipoproteins, such as acetylated LDL (AcLDL) and oxidized LDL (OxLDL), and often many other types of ligands (1-3). Initially, scavenger receptor activity was identified as "AcLDL receptor activity" by Brown, Goldstein, and colleagues (2), who were developing an in vitro model for the deposition of LDL cholesterol in macrophages in artery walls during the initial stages of atherosclerotic plaque formation. They discovered that acetylation of LDL redirected the uptake of the particles from classic LDL receptors to a novel AcLDL receptor activity, which appeared to be relatively macrophage-specific. The massive, receptor-mediated uptake of cholesterol from modified LDL can convert cultured macrophages into cholesteryl ester-filled foam cells, similar to those in atherosclerotic plaques. As a consequence, investigators interested in lipoprotein metabolism and atherosclerosis have focused considerable attention on these receptors.

Brown and Goldstein also showed that these macrophage receptors mediate the binding of a remarkably wide variety of polyanionic ligands (e.g., modified proteins, sulfated polysaccharides, and certain polynucleotides; reviewed in refs. 2-4). Such broad binding specificity prompted the name change to "scavenger receptor" and the proposal $(1,3)$ that these receptors participate in the innate immune system by serving as pattern recognition receptors (5) that bind a wide variety of components of pathogens (1). Such recognition is a prerequisite to mounting cellular and/or humoral responses to protect the body. Current data suggest that SRs can participate in innate immunity $(1,4,6)$. With respect to their broad ligand specificities and their likely role in protecting the host, SRs are similar to hepatic cytochrome P450s. The broad and overlapping substrate specificities that characterize that large family of enzymes allow the liver to inactivate a wide variety of potentially toxic small molecules. By analogy, it seemed likely that there would be multiple classes of scavenger receptors with overlapping specificities to permit the recognition of many different potentially pathogenic structures (1) and that SRs would have arisen early in evolution to allow multicellular organisms to recognize a multitude of endogenous or exogenous structures (1). Indeed, over the past decade, the cDNAs for at least nine distinct scavenger receptors have been cloned and analyzed from organisms as diverse as mammals and fruit flies. These receptors have been categorized into broad classes (A, B, C, etc.) based on global structural similarities. In many cases, the members of a given class have been subdivided into "types" based on more subtle structural differences, including multiple proteins from a single gene generated by alternative RNA splicing (1). The class A, type I and II scavenger receptors (SR-AI/II), the first SRs to be identified and cloned (3), are the subject of the article by Platt and Gordon in this Perspective series (4). Here, I will consider the class B, type I scavenger receptor, SR-BI, and studies that have refocused interest in scavenger receptors on lipoprotein metabolism.

\section{SR-BI binds HDL and mediates \\ selective lipid uptake}

SR-BI was initially identified in a scavenger receptor expression cloning study that used AcLDL as the ligand (7). SR-BI is a 509-amino-acid-long member of the CD36 superfamily of proteins. The SR-BI identified in rodents is an ortholog of (essentially identical to) human CLA-1 (now also called hSR-BI), whose cDNA was independently cloned as a homolog of CD36 with unknown function (8). In addition to CD36, another class B scavenger receptor (refs. 7, 9; see also Febbraio et al., this Perspective series, ref. 10), the CD36 family members (reviewed in refs. 10,11) include lysosomal integral membrane protein II (LIMP-II; a lysosomal protein), croquemort (a Drosopbila hemocyte/macrophage apoptotic cell receptor), and SnmP-1 (a silk moth olfactory neuron membrane protein). Most members of the CD36 superfamily share about $30 \%$ sequence identity. They have been proposed to have horseshoe-like membrane topologies (Figure 1) with short $\mathrm{N}$ - and C-terminal cytoplasmic domains, adjacent $\mathrm{N}$ - and $\mathrm{C}$-terminal transmembrane domains, and the bulk of the protein in a heavily $\mathrm{N}$-glycosylated, disulfide-containing extracellular loop. There are alternatively spliced mRNAs for both CD36 $(10,11)$ and SR-BI (ref. 12; alternative form 


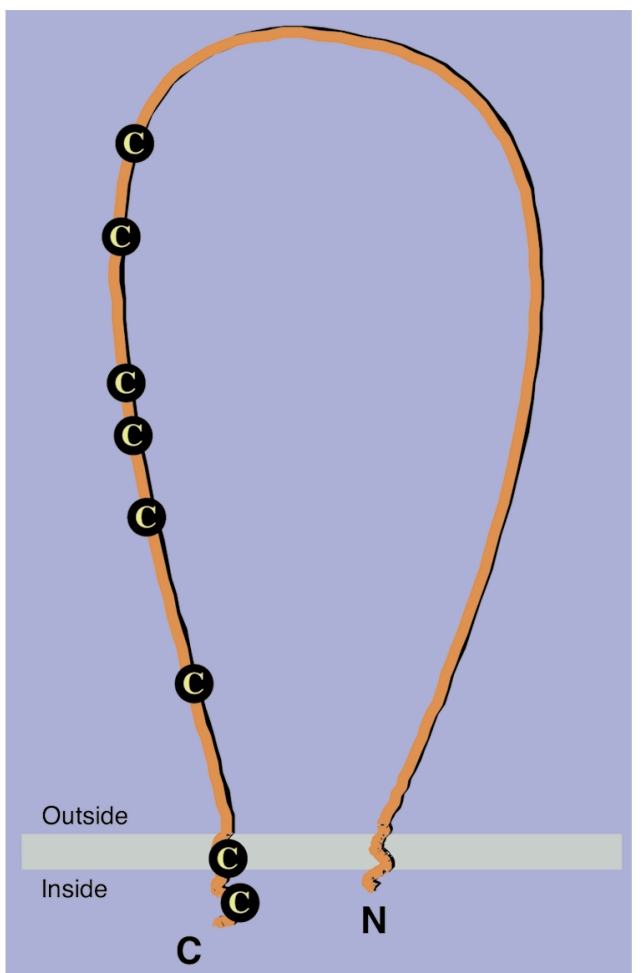

Figure 1

Model of the topology of SR-BI. SR-BI is a 509-residue glycoprotein with a large extracellular loop ( $\sim 403$ residues) anchored to the plasma membrane at both the $\mathrm{N}$ - and $\mathrm{C}$-termini by transmembrane domains ( 28 and $\sim 25$ residues) which have short extensions into the cytoplasm ( $~ 8$ $\mathrm{N}$-terminal residues and $\sim 45 \mathrm{C}$-terminal residues). The approximate locations of the cysteines are shown ("C"). The protein is heavily N-glycosylated, and it is palmitoylated on the cysteines in the C-terminal cytoplasmic and transmembrane domains, $\mathrm{Cys}^{462}$ and $\mathrm{Cys}^{470}$ (murine numbering system). Adapted from ref. 11.

designated SR-BII), and both are fatty acylated proteins that cluster in caveola-like cholesterol-rich lipid domains in cultured cells (see Febbraio et al., this Perspective series, ref. 10; see also ref. 11).

Shortly after SR-BI was cloned, it was shown to bind to a variety of ligands other than AcLDL, including OxLDL, maleylated BSA, anionic phospholipids, apoptotic cells, and unmodified LDL and VLDL (11). The most striking and unexpected finding was that SR-BI binds HDL with high affinity $(11,13)$, raising the possibility, now confirmed, that SR-BI represented a longsought physiologically relevant HDL receptor.

As is the case for the classic LDL receptor (LDLR), SRBI facilitates the cellular uptake of cholesterol (primarily in the form of cholesteryl esters) from the hydrophobic cores of lipoproteins by first mediating the binding of the lipoprotein to the outer surfaces of the cells. However, the mechanism of lipid uptake following lipoprotein binding for SR-BI differs markedly from that of the LDLR. The LDLR mediates endocytosis of the intact LDL particle via coated pits and vesicles, and its subsequent hydrolysis in lysosomes (14). SR-BI mediates the selective uptake of HDL's cholesteryl esters $(11,13)$. Selective uptake involves efficient transfer to cells of the cholesteryl esters from the lipoprotein's hydrophobic core, but not the apolipoprotein at the lipoprotein's surface. It does not involve the sequential internalization of the intact lipoprotein particle and its subsequent degradation. Selective lipid uptake in vivo, primarily by the liver and steroidogenic tissues, was first identified almost 20 years ago by Glass et al. and Stein et al. during tissue clearance studies of plasma HDL differentially radiolabeled on both its lipid and protein components (reviewed in ref. 11).

SR-BI-mediated selective lipid uptake appears to be a two-step process, in which high-affinity lipoprotein binding is followed by receptor-mediated transfer of lipid from the lipoprotein particle to the cell membrane (reviewed in ref. 11). After lipid transfer, the lipiddepleted lipoprotein particle is released from the cells and re-enters the extracellular space. SR-BI can also mediate the bidirectional flux of unesterified cholesterol and phospholipids between HDL and cells, although the physiologic significance of SRBI-dependent cellular cholesterol efflux has not been established. SR-BI can function as an LDL receptor (binding and selective uptake) as well as an HDL receptor (reviewed in ref. 11). CD36 can also bind HDL and LDL, but it cannot efficiently mediate cholesteryl ester uptake (reviewed in ref. 11). The detailed molecular mechanism underlying SR-BI-mediated selective lipid uptake has not yet been elucidated.

Several techniques have been used to show that there are distinct modes of binding and perhaps distinct binding sites for LDL and HDL on SR-BI $(11,15,16)$. Strikingly, HDL competes effectively for the binding of LDL to SR-BI, whereas LDL can only partially compete for HDL binding to SR-BI (13). This phenomenon, termed "nonreciprocal cross-competition" (NRCC), has been documented in studies of SR-AI and SR-AII as well (17). In NRCC, one ligand efficiently competes for the binding of a second ligand whereas the second ligand fails to compete, or competes only partially, for the binding of the first. The observation of NRCC between ligands probably indicates that the receptor carries multiple binding sites with differing ligand binding properties. NRCC might also be observed under physiologically relevant experimental conditions (or, presumably, in vivo conditions) if ligand binding does not proceed to equilibrium. However it occurs, NRCC could cause the preferential binding of one ligand out of a complex mix of available ligands. Indeed, as a consequence of NRCC, the preferred ligand need not be the one with the tightest equilibrium binding (lowest $K_{\mathrm{d}}$ ). For example, in the case of SR-BI, HDL does not bind as tightly as LDL, yet HDL nevertheless binds preferentially when both ligands are present $(11,13,15)$. Thus, because of NRCC (3), LDL is unlikely to substantially block SR-BI's binding of HDL in vivo. The molecular bases for NRCC by SR-BI are currently under study.

\section{SR-BI is a physiologically relevant HDL receptor} Three kinds of primarily in vivo studies using mainly mice and rats have established that SR-BI is a physiologically relevant HDL receptor. First, the tissue distribution of SR-BI is that expected for an HDL receptor 
$(11,13)$. It is highly expressed in liver and steroidogenic tissues, precisely those tissues that exhibit the highest levels of selective uptake of HDL cholesteryl esters. It is also expressed at lower levels in a variety of other tissues and cell types at various stages of development and adulthood. These include the apical surfaces of absorptive cells in the proximal small intestine, the mammary glands of pregnant rodents, embryo proximal decidual cells of the uterus, trophoblasts and yolk sac visceral endoderm during embryonic development, and macrophages in atherosclerotic plaques $(11,16,18)$.

Second, SR-BI expression is coordinately regulated with cholesterol metabolism. Its expression in steroidogenic cells in vivo and in vitro is regulated by trophic hormones, apparently via the cAMP/protein kinase A signal transduction pathway that includes transcription factors such as CCAAT/enhancer-binding proteins (C/EBPs) and steroidogenic factor-1 (SF-1) (reviewed in refs. 11, 16). For example, in vivo adrenocortical SR-BI expression and steroidogenesis are stimulated by systemic administration of adrenocorticotropic hormone and inhibited by dexamethasone. Human chorionic gonadotropin stimulates testicular Leydig cell steroidogenesis and SR-BI expression. SR-BI expression in hepatocytes, steroidogenic cells, or macrophages can be altered by administration of estrogen (a speciesdependent phenomenon), PPAR agonist, vitamin E, polyunsaturated fatty acids, cholesterol, and other agents $(11,16,19,20)$. Intestinal SR-BI expression is also regulated. When the composition (phospholipids, bile acids, cholesterol) and/or amounts of bile are altered by surgical bile diversion or bile duct ligation or by mutation of the Mdr2 or Cyp7a genes, SR-BI expression changes coordinately with cholesterol absorption (21). The transcriptional regulation of SR-BI appears to be due to $S R-B I$ promoter binding sites for a number of transcription factors (reviewed in refs. 11, 16), including C/EBP, SF-1, and sterol regulatory element binding protein-1 (SREBP-1). In addition, Ikemoto and colleagues have identified a PDZ domain-containing cytosolic protein that interacts with the $\mathrm{C}$-terminus of SR-BI and may influence its activity (22).

Third, alteration of SR-BI activity in vitro (e.g., in the presence of blocking antibodies; ref. 23) or in vivo (e.g., in transgenic or knockout [KO] mice; reviewed in refs. 11, 16; see also refs. 24-29) has a corresponding effect on cholesterol metabolism. For example, hepatic overexpression is accompanied by decreased plasma levels of HDL cholesterol and increased biliary cholesterol, but not bile acid or phospholipid, content. This is consistent with a model in which hepatic SR-BI mediates the transfer of cholesterol from plasma HDL to the bile for excretion (Figure 2). In SR-BI-null KO mice (24), plasma total cholesterol is elevated approximately twofold, and most of this material circulates in abnormally large, heterogeneous, apoE-enriched HDL-like particles. This finding provides strong evidence of a role for SR-BI-mediated selective lipid uptake in cholesterol clearance from the plasma. There is also a marked reduction in the cholesteryl ester stores in steroidogenic tissues of SR-BI KO mice relative to controls. SR-BI deficiency also reduces biliary cholesterol secretion without altering biliary bile acid secretion, bile acid pool size, or fecal bile excretion $(24,28,30)$. Thus, SR-BI plays a key role in controlling the structure and amount of cholesterol in plasma HDL, steroidogenic and hepatic uptake of HDL cholesterol, and the use of HDL cholesterol for biliary cholesterol secretion. Figure 2 illustrates the proposed roles of HDL and SR$\mathrm{BI}$ in mediating the transport of cholesterol from peripheral tissues to the liver for excretion into the bile, a process called "reverse cholesterol transport."

\section{SR-BI and atherosclerosis}

In humans and some animal models, levels of plasma HDL cholesterol (the "good" cholesterol) are inversely correlated with risk for, or severity of, atherosclerosis (11). Thus, the following question arises: Is hepatic SRBI expression antiatherogenic because it increases reverse cholesterol transport and thus removal of cholesterol from the body, or might it favor atherosclerosis by decreasing total plasma HDL cholesterol? Although we don't yet know the answer for humans, the answer for murine models of atherosclerosis is unequivocal. The murine atherosclerosis models reported to date have been the apoE KO mouse and the high-fat-/high-cholesterol-fed LDLR KO mouse, which serves as a model for human familial hypercholesterolemia (14). The absence of SR-BI in KO mice dramatically accelerates the onset of atherosclerosis $(28,29)$, whereas atherosclerosis is suppressed by hepatic overexpression of SR-BI (25-27). The antiatherogenic effects of increased hepatic SR-BI expression raise the possibility that SR-BI could be targeted for pharmacologic or genetic therapy to treat the most common cause of death in industrialized nations, cardiovascular disease.

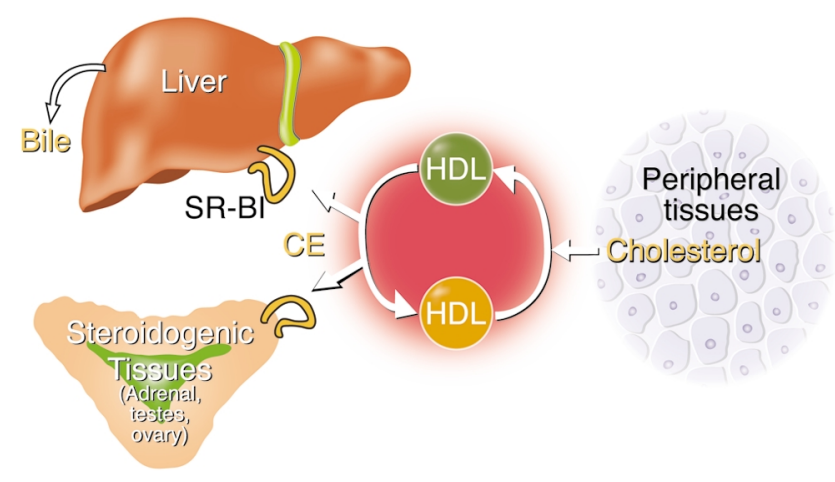

\section{Figure 2}

Role of SR-BI in HDL metabolism in vivo. HDL is thought to extract cellular cholesterol from peripheral tissues by a mechanism involving the product of the $A B C A 1$ (Tangier disease) gene. After the plasma HDL-cholesterol (HDL-C) is esterified to cholesteryl ester (CE) by the enzyme lipoprotein lipase (not shown), it can be transported to the liver by either an indirect pathway (transfer to other lipoproteins followed by hepatic receptor-mediated endocytosis, not shown) or a direct pathway via SR-BI and selective cholesterol uptake. The HDL-C in the liver can be secreted into the bile, either as cholesterol or as bile acids. The delivery of cholesterol from peripheral tissues via plasma HDL to the liver for biliary excretion as cholesterol or bile acids is called "reverse cholesterol transport." SR-BI also can mediate HDL-C uptake by steroidogenic tissues for steroid hormone synthesis or cholesterol storage. Redrawn from refs. 11, 34. 


\section{SR-BI and intestinal cholesterol absorption}

The expression of SR-BI on the apical surface of intestinal epithelial cells and in vitro cholesterol transport assays in brush border membranes raised the possibility that SR-BI plays a critical role in cholesterol absorption, perhaps serving as the long-sought cholesterol transporter (18). Although the regulation of intestinal SR-BI described above is consistent with this suggestion, intestinal cholesterol absorption is not reduced and fecal sterol excretion is not increased in SR-BI KO mice relative to controls (30). Thus, SR-BI expression is not essential for intestinal cholesterol absorption. It remains possible that SR-BI normally participates either directly or indirectly in intestinal cholesterol absorption. However, if it normally plays an important role in cholesterol absorption, there must be very efficient compensatory mechanisms that allow absorption in the SR-BI KO mouse.

\section{SR-BI and other physiologic systems}

Given the importance of HDL for the transport of lipids, including cholesterol and fat-soluble vitamins, in mammals (especially rodents), it is not surprising that the SR$\mathrm{BI}$ deficiency in SR-BI KO mice causes abnormalities in several distinct physiologic systems. Two striking cases are the development of oocytes in the female reproductive system and red blood cell development.

First, female - but not male - SR-BI KO mice are infertile (28). The female infertility is not due simply to depletion of ovarian cholesterol stores; the ovaries of SR-BI KO females can produce normal amounts of plasma progesterone to support pregnancy (28). Furthermore, SR-BI KO females exhibit no obvious defects in gross ovarian morphology or in their estrus cycles, and they ovulate normal numbers of oocytes. The ovulated oocytes, however, are dysfunctional. A significant fraction of the ovulated oocytes die soon after ovulation, and all preimplantation embryos from SR-BI KO females isolated the morning after mating either are dead or die soon afterwards. Thus, SR-BI can either directly or indirectly dramatically influence oocyte development. The mechanism underlying the infertility is currently under study using genetic and pharmacologic methods. The abnormal structure, composition, and/or abundance of lipoproteins in the SR-BI KO females apparently contribute to the infertility $(\mathrm{H}$. Miettinen et al., unpublished data).

Second, SR-BI KO mice exhibit a disruption in the late stages of erythroid differentiation (T.M. Holm et al., unpublished data). Erythrocytes from these animals are morphologically abnormal, especially when their diet or their genetic background favors hypercholesterolemia. Erythrocytes in SR-BI/apoE double $\mathrm{KO}$ mice are anucleate and contain approximately normal amounts of hemoglobin, but they exhibit the features of proposed intermediates in reticulocyte differentiation, including macrocytosis, irregular shape, and large autophagosomes. Erythrocytes from apoE KO mice with normal SR-BI exhibit no such abnormalities. Incubation of SR-BI/apoE double KO erythrocytes in normolipidemic serum leads to expulsion of the autophagosomes. Thus, SR-BI-defi- cient mice exhibit a defect in erythroid maturation that may prove useful for the detailed analysis of the final steps in reticulocyte differentiation.

\section{Questions arising}

One might reasonably expect a multiligand scavenger receptor that binds anionic phospholipids and modified proteins to be involved in pattern recognition for host defense and/or removal of senescent/apoptotic cells $(1,31)$. Unexpectedly, one scavenger receptor, SR$\mathrm{BI}$, also functions as an HDL receptor that plays a key role in lipid (cholesterol) metabolism. Its homolog CD36, another multiligand class B scavenger receptor, also plays a role in lipid (fatty acid) metabolism, adhesion, and the phagocytosis of apoptotic neutrophils by macrophages and the phagocytosis of rod outer segments by retinal pigment epithelium (10). These two class B scavenger receptors share many features in common (caveola-like domain clustering, many shared ligands), yet they differ greatly in tissue distribution, ability to mediate selective cholesterol uptake, and other critical physiologic functions. It is possible that other, as-yet unrecognized, physiologic activities depend on these receptors. How many of the in vitro activities attributed to these multiligand receptors have corresponding in vivo functions? Why is another lipoprotein receptor, the LDL receptor-related protein (LRP), also a multiligand receptor with multiple functions (see ref. 3; see also Herz and Strickland, this Perspective series, ref. 32)? Did multiligand lipoprotein receptors such as SR-BI and LRP evolve from pattern recognition receptors for host defense before the emergence of more ligand-specific lipoprotein receptors (e.g., LDLR)? In some cases, multifunctionality may simply reflect functional "moonlighting" - one protein performing multiple independent functions (33) - but there may also be deeper physiologic or evolutionary relationships underlying the complex properties of these multiligand, multifunctional receptors.

\section{Acknowledgments}

I am grateful to the many colleagues and collaborators (too numerous to list here) who have contributed to the analysis of scavenger receptor structure and function. Because of editorial limitations, it has been necessary to cite indirectly via reviews, rather than to cite primary publications directly. I appreciate the understanding of those investigators whose important contributions have been cited indirectly. I am especially grateful to my collaborators who have permitted me to discuss some of our work prior to publication. None of the work from my laboratory would have been possible without the ongoing support of the NIH Heart, Lung, and Blood Institute.

\footnotetext{
1. Krieger, M. 1997. The other side of scavenger receptors: pattern recognition for host defense. Curr. Opin. Lipidol. 8:275-280.

2. Brown, M.S., and Goldstein, J.L. 1983. Lipoprotein metabolism in the macrophage: implications for cholesterol deposition in atherosclerosis. Annu. Rev. Biochem. 52:223-261.

3. Krieger, M., and Herz, J. 1994. Structures and functions of multiligand lipoprotein receptors: macrophage scavenger receptors and LDL receptor-related protein (LRP). Annu. Rev. Biochem. 63:601-637.

4. Platt, N., and Gordon, S. 2001. Is the class A macrophage scavenger recep-
} 
tor (SR-A) multifunctional? - The mouse's tale. J. Clin. Invest. 108:649-654.

5. Janeway, C.A. 1989. Approaching the asymptote? Evolution and revolution in immunology. Cold Spring Harb. Symp. Quant. Biol. 54:1-13.

6. Pearson, A.M. 1996. Scavenger receptors in innate immunity. Curr. Opin. Immunol. 8:20-28.

7. Acton, S.L., Scherer, P.E., Lodish, H.F., and Krieger, M. 1994. Expression cloning of SR-BI, a CD36-related class B scavenger receptor. J. Biol. Chem. 269:21003-21009.

8. Calvo, D., and Vega, M.A. 1993. Identification, primary structure, and distribution of CLA-1, a novel member of the CD36/LIMPII gene family. J. Biol. Chem. 268:18929-18935.

9. Endemann, G., et al. 1993. CD36 is a receptor for oxidized low density lipoprotein. J. Biol. Chem. 268:11811-11816.

10. Febbraio, M., Hajjar, D.P., and Silverstein, R.L. 2001. CD36: a class B scavenger receptor involved in angiogenesis, atherosclerosis, inflammation, and lipid metabolism. J. Clin. Invest. 108:785-791.

11. Krieger, M. 1999. Charting the fate of the "good cholesterol": identification and characterization of the high-density lipoprotein receptor SR-BI. Annu. Rev. Biochem. 68:523-558.

12. Webb, N.R., et al. 1998. SR-BII, an isoform of the scavenger receptor BI containing an alternate cytoplasmic tail, mediates lipid transfer between high density lipoprotein and cells. J. Biol. Chem. 273:15241-15248.

13. Acton, S., et al. 1996. Identification of scavenger receptor SR-BI as a high density lipoprotein receptor. Science. 271:518-520.

14. Goldstein, J.L., Brown, M.S., Anderson, R.G., Russell, D.W., and Schneider, W.J. 1985. Receptor-mediated endocytosis: concepts emerging from the LDL receptor system. Annu. Rev. Cell. Biol. 1:1-39.

15. Gu, X., Lawrence, R., and Krieger, M. 2000. Dissociation of the high density lipoprotein and low density lipoprotein binding activities of murine scavenger receptor class B type I (mSR-BI) using retrovirus library-based activity dissection. J. Biol. Chem. 275:9120-9130.

16. Trigatti, B., Rigotti, A., and Krieger, M. 2000. The role of the high-density lipoprotein receptor SR-BI in cholesterol metabolism. Curr. Opin. Lipidol. 11:123-131.

17. Ashkenas, J., Penman, M., Vasile, E., Freeman, M., and Krieger, M. 1993. Structures and high and low affinity ligand binding properties of murine type I and type II macrophage scavenger receptors. J. Lipid Res. 34:983-1000.

18. Hauser, H., et al. 1998. Identification of a receptor mediating absorption of dietary cholesterol in the intestine. Biochemistry. 37:17843-17850.

19. Spady, D.K., Kearney, D.M., and Hobbs, H.H. 1999. Polyunsaturated fatty acids up-regulate hepatic scavenger receptor B1 (SR-BI) expression and HDL cholesteryl ester uptake in the hamster. J. Lipid Res. 40:1384-1394.

20. Witt, W., Kolleck, I., Fechner, H., Sinha, P., and Rustow, B. 2000. Regula- tion by vitamin $\mathrm{E}$ of the scavenger receptor $\mathrm{BI}$ in rat liver and HepG2 cells J. Lipid Res. 41:2009-2016.

21. Voshol, P.J., et al. 2001. Down-regulation of intestinal scavenger receptor class B, type I (SR-BI) expression in rodents under conditions of deficient bile delivery to the intestine. Biochem. J. 356:317-325.

22. Ikemoto, M., et al. 2000. Identification of a PDZ-domain-containing protein that interacts with the scavenger receptor class B type I. Proc. Natl. Acad. Sci. USA. 97:6538-6543.

23. Temel, R.E., et al. 1997. Scavenger receptor class B, type I (SR-BI) is the major route for the delivery of high density lipoprotein cholesterol to the steroidogenic pathway in cultured mouse adrenocortical cells. Proc. Natl. Acad. Sci. USA. 94:13600-13605.

24. Rigotti, A., et al. 1997. A targeted mutation in the murine gene encoding the high density lipoprotein (HDL) receptor scavenger receptor class B type I reveals its key role in HDL metabolism. Proc. Natl. Acad. Sci. USA. 94:12610-12615

25. Arai, T., Wang, N., Bezouevski, M., Welch, C., and Tall, A.R. 1999. Decreased atherosclerosis in heterozygous low density lipoprotein receptor-deficient mice expressing the scavenger receptor BI transgene. J. Biol. Chem. 274:2366-2371.

26. Ueda, Y., et al. 2000. Relationship between expression levels and atherogenesis in scavenger receptor class B, type I transgenics. J. Biol. Chem 275:20368-20373.

27. Kozarsky, K.F., Donahee, M.H., Glick, J.M., Krieger, M., and Rader, D.J 2000. Gene transfer and hepatic overexpression of the HDL receptor SR$\mathrm{BI}$ reduces atherosclerosis in the cholesterol-fed LDL receptor-deficient mouse. Arterioscler. Thromb. Vasc. Biol. 20:721-727.

28. Trigatti, B., et al. 1999. Influence of the high density lipoprotein receptor SR-BI on reproductive and cardiovascular pathophysiology. Proc. Natl. Acad. Sci. USA. 96:9322-9327.

29. Huszar, D., et al. 2000. Increased LDL cholesterol and atherosclerosis in LDL receptor-deficient mice with attenuated expression of scavenger receptor B1. Arterioscler. Thromb. Vasc. Biol. 20:1068-1073.

30. Mardones, P., et al. 2001. Hepatic cholesterol and bile acid metabolism and intestinal cholesterol absorption in scavenger receptor class B type Ideficient mice. J. Lipid Res. 42:170-180.

31. Bird, D.A., et al. 1999. Receptors for oxidized low-density lipoprotein on elicited mouse peritoneal macrophages can recognize both the modified lipid moieties and the modified protein moieties: implications with respect to macrophage recognition of apoptotic cells. Proc. Natl. Acad. Sci. USA. 96:6347-6352.

32. Herz, J., and Strickland, D.K. 2001. LRP: a multifunctional scavenger and signaling receptor. 108:779-784.

33. Jeffery, C.J. 1999. Moonlighting proteins. Trends Biochem. Sci. 24:8-11.

34. Rigotti, A., and Krieger, M. 1999. Getting a handle on "good" cholesterol with the high-density lipoprotein receptor. N. Engl.J. Med. 341:2011-2013. 